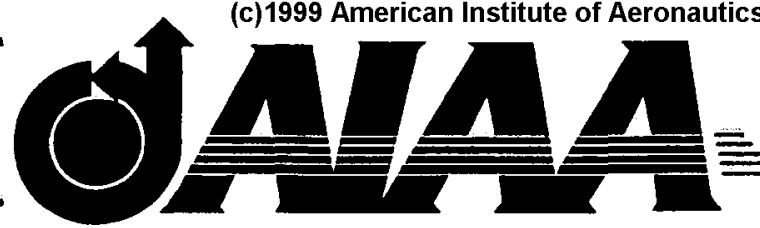

\title{
AIAA 99-3451
}

DSMC Modeling of Vibration-Translation Energy Transfer in Hypersonic Rarefied Flows

S.F. Gimelshein

George Washington University,

Washington, DC 20052

I.D. Boyd and Q. Sun

University of Michigan,

Ann Arbor, MI 48109

M.S. Ivanov

Institute of Theoretical and Applied Mechanics

Novosibirsk 630090, Russia

\section{3rd Thermophysics Conference 28 June - 1 July, 1999 / Norfolk, VA}

For permission to copy or republish, contact the American Institute of Aeronautics and Astronautics 1801 Alexander Bell Drive, Suite 500, Reston, VA 22091 


\title{
DSMC Modeling of Vibration-Translation Energy Transfer in Hypersonic Rarefied Flows
}

\author{
S.F. Gimelshein* \\ George Washington University \\ Washington, DC 20052 \\ I.D. Boyd ${ }^{\dagger}$ and Q. Sun ${ }^{\ddagger}$ \\ University of Michigan \\ Ann Arbor, MI 48109 \\ M.S. Ivanov ${ }^{5}$ \\ Institute of Theoretical and Applied Mechanics \\ 630090 Novosibirsk, Russia
}

\begin{abstract}
The influence of the vibration-translation energy transfer model on a flow about a sphere in the transitional and near-continuum regimes is examined using the direct simulation Monte Carlo method. Both the macroparameters and vibrational distribution functions in different regions of the flow are studied. Four different models of vibration-translation exchanges suitable for particle simulation are considered. Also, the impact of vibration-vibration energy transfer is investigated. The results showed a significant sensitivity of vibrational temperatures, and especially vibrational populations, to the choice of vibrational model, while most of the macroparameters are not considerably impacted by changing the model.
\end{abstract}

\section{Introduction}

Practical needs connected with hypersonic flights of space vehicles at high altitudes $(H>80 \mathrm{~km})$ demand the investigation of rarefaction phenomena inherent to

\footnotetext{
- Research scientist, Department of Chemistry. E-mail gimel@gwu.edu.

$\uparrow$ Associate Professor, Aerospace Engineering. Member AlAA. E-mail iainboyd@engin.umich.edu.

t Research Associate, Aerospace Engineering.

5 Head, Computational Aerodynamics Laboratory. Senior Member AlAA. E-mail ivanov@itam.nsc.ru

Copyright (C)1999 by I.D. Boyd, Q. Sun, S.F. Gimelshein, M.S. Ivanov. Published by the American Institute of Aeronautics and Astronautics, Inc. with permission
}

the low density environment. Important effects include physiochemical phenomena that occur in the high temperature region behind the bow shock. The main tool for investigating these rarefied chemically reacting flows is the direct simulation Monte Carlo (DSMC) method [1].

A number of models suitable for the DSMC method have been developed lately that take into account real gas effects in high-temperature hypersonic flows, i.e. the excitation of vibrational degrees of freedom and chemical reactions. For modeling vibration-translation (VT) energy transfer, several level-by-level models are suggested in the literature, different in their theoretical ground and numerical efficiency. Nevertheless, up to now the most frequently used model is the simplified Larsen-Borgnakke (LB) model, where post-collision energies are assumed to be distributed according to the local equilibrium distribution functions. Formerly constructed for continuous internal energy spectra, it was extended to the case of discrete energies, but did not lose its phenomenological ground.

Meanwhile, results of DSMC computations of rarefied hypersonic flows might be strongly influenced by the models of VT energy transfer used in the computations. The use of the conventional LB model may significantly bias the results. From the other hand, the use of a sophisticated level-by-level model would require additional computer time and/or memory. It is important therefore to know how the model of energy transfer influences chemically reacting rarefied flows, and to determine the area of applicability of different models.

The objective of the present paper is to investigate different models of VT energy transfer for the DSMC 
method, and to examine the impact of the model on high-temperature flow. Based on the kinetic description of gas flows, the DSMC method provides information both on gas macroparameters, such as density, temperature, etc., and particle velocity and energy distribution functions. The latter might be of great importance for understanding physical peculiarities of hypersonic rarefied flows where chemical reactions and energy transfer between the vibrational, rotational and translational (VRT) modes of gas molecules take place. Therefore, special attention is paid in this work to a detailed analysis of the distribution functions of vibrational energy. The importance of such an analysis becomes evident from the consideration that the vibrational distribution may strongly affect the rate of important chemical reactions.

\section{Numerical technique}

Two computational systems based on the DSMC method, MONACO and SMILE, have been used in this work. The structure and main features of the systems were described in detail in [1] and [2], respectively. Here we mention the principal differences between them.

- Cell structure: body-fitted triangular grid is utilized in MONACO, whereas SMILE uses a two-level rectangular grid.

- Grid adaption: mean free path based adaption in MONACO and automatic density gradient adaption based in all space directions in SMILE.

- Macroparameter grid: MONACO uses a sub-cell scheme for selection of collision partners, while SMILE utilizes a grid separated from that used for collisions.

- Collision scheme: cell scheme with a sub-cell capability in MONACO and combined cell/free cell technique [3] in SMILE.

Meantime, the two systems make use of the same collision models: intermolecular interaction is controlled with the VHS/VSS model, the models described above for rotational and vibrational energies are used in the energy transfer, Maxwell model is employed for gas/surface interaction. The two systems make use of collision grids adaptive to the flow gradients. Both of them are suitable to modern parallel computers, and the use of special techniques reducing computational cost such as particle doubling or space or species weighting is possible thercin. All this therefore creates a basis for a. good compatibility between the systems.

\section{Vibration-translation energy transfer models}

This section gives a summary of models used to simulate vibration-translation energy exchange. We give here only a short overview, since the conplete information on the models can be found elsewhere $[4,5,6]$.

\subsection{Phenomenological modeling}

The first model (below Model 1) discussed here employs a phenomenological approach. Its detailed description may be found in [4]. It employs the Borgnakke-Larsen (B-L) scheme for energy re-distribution that is widely used in DSMC computations. The current implementation of this model is formulated to employ molecular quantities (as opposed to temperature) to control the rate of relaxation. The instantaneous inelastic collision probability is written as

$$
\begin{gathered}
\phi(g)=\frac{\dot{\phi_{L T}} \phi_{P}}{\phi_{L T}+\phi_{P}} \\
\phi_{L T}(g)=\frac{1}{Z_{0}} g^{\alpha} \exp \left(\frac{-g^{*}}{g}\right),
\end{gathered}
$$

where $g$ is the relative collision velocity, and the constants $\alpha, g^{*}$, and $Z_{0}$ are related to the known properties of colliding molecules. These constants are chosen to satisfy Millikan and White [7] results. $\phi_{P}$ is the hightemperature correction [8].

In order to satisfy the detailed halance principle, the B-L equilibrium distribution function for sampling vibrational energy is modified in accordance with [9] as follows:

$$
f_{A b e}=\frac{\phi\left(g^{\prime}\right)}{\phi_{\max }} * f_{B-L}
$$

Here, $g^{\prime}$ is the post-collision relative velocity consistent with the sampled value of vibrational energy.

Finally, to match the DSMC and continuum values of VT exchange average probabilities, the correction [10] is used. This correction requires the instantaneous collision probability to be multiplied by the factor $1+\zeta_{V} /(4-2 \omega)$, where $\zeta_{V}$ is the number of degrees of freedom of the vibrational energy mode and $\omega$ defines the intermolecular interaction in the VHS collision model.

\subsection{Multiple quantum-step transition model}

The second model (Model 2), the Multiple QuantumStep Transition model [4], first introduced in [11], employs a more detailed approach to modeling vibrational relaxation. The vibrational energy is characterized by 
the bounded harmonic oscillator assumption. The probability of activation from energy level $v$ by $\Delta$ is

$P_{v, v+\Delta}=v !(v+\Delta) ! e^{-\epsilon_{1}} \epsilon_{1}^{2 v+\Delta}\left|\sum_{k=0}^{v} \frac{(-1)^{k} \epsilon_{1}^{-k}}{k !(v-k) !(v+\Delta-k) !}\right|^{2}$

Here, the quantity $\epsilon_{1}$ is

$$
\epsilon_{1}=a e^{-g / g},
$$

and $g^{*}$ and $a$ are constants. To satisfy strictly the detailed balance requirement, the deactivation probability is written as

$$
P_{v+\Delta, v}=\frac{\left(g^{2}+g^{2}\right)^{1-\omega}}{g^{2-2 \omega}} P_{v, v+\Delta}\left(\sqrt{g^{2}+g^{\prime 2}}\right)
$$

Computation of transition probabilities for each collision in a DSMC simulation is very expensive numerically. To reduce this cost, a look-up table is generated. This table consists of activation and deactivation probabilities referenced by relative velocity of collision, vibrational energy level, desired number of quantum-steps for transition and the species type. This study used a table with 300 evenly spaced values for relative velocity and a transition of up to 5 quantum-steps. The maximum value of relative velocity included in the table was $20,000 \mathrm{~m} / \mathrm{s}$.

\subsection{Quasiclassical energy transfer model}

The model [5] (Model 3 hereafter) uses the results of the approach based on a new variant of the quasiclassical multidimensional scattering theory for the description of the vibration-rotation-translation exchange of polyatomic molecules [12]. The anharmonicity effects are included, and the multi-quantum transition probabilities are obtained automatically without a compilation of different approximations.

The energy-dependent form for VT transition crosssections calculated in the framework of the quasiclassical theory was obtained using a mean frequency approximation. That implies the arithmetic average of initial and final states, both internal and translational energies, to be taken into consideration. This allows one to simplify the expressions for the correspondent transition cross sections derived by the use of generalized eikonal approximation.

Depending on the relative collision velocity, all collisions are classified into three groups, slow, intermediate, and fast. Complicated integral expressions are used for computing cross sections of slow and fast collisions (omitted here, they may be found in [5]), and a simple exponential interpolation of these two expressions is utilized for intermediate collisions. The VT probabilities are found then as the ratio of the calculated VT cross section to the total collision cross section. As the expressions for transition probabilities are fairly cumbersome, the look-up tables are used in DSMC computations.

\subsection{Nonperturbative semiclassical model}

The fourth model used in this work is a threedimensional semiclassical model (Model 4) developed recently by Adamovich and Rich [6] for collisions between rotating diatomic molecules. The energy transfer probabilities in this model are evaluated using the nonperturbative forced harmonic oscillator model. The model provides for the probabilities of VT energy transfer as functions of the total collision energy, orientation of a molecule during a collision, its rotational energy, and impact parameter. The look-up table was calculated for this model to be referred to in DSMC computations.

\subsection{VV energy transfer}

The model [5] is used in this work. The derivation of the model is similar to that of Model 3 for VT energy transfer, and is also based on the quasiclassical multidimensional scattering theory [12]. The mean frequency approximation was used for obtaining energydependent probabilities of VV level-to-level transitions. Only generalized isoquantum transitions are allowed in this model. The detailed explanation of the model is beyond the scope of this paper, and may be found in [5]. We note here only that look-up tables were used in simulations, similar to those for the VT models.

\section{Flow conditions and model pa- rameters}

The nitrogen flow about a sphere of radius $10 \mathrm{~cm}$ is considered in this work. The free stream velocity was assumed to be $5,100 \mathrm{~m} / \mathrm{s}$, and the free-stream temperature was $190 \mathrm{~K}$. Two values of the free stream number density were considered, $1.2 \times 10^{21}$ molecules $/ \mathrm{m}^{3}$ and $1.2 \times 10^{22}$ molecules $/ \mathrm{m}^{3}$, which gives a Knudsen number $K n$ of 0.01 and 0.001 , respectively, based on the radius of the sphere. A wall temperature of $500 \mathrm{~K}$ was used.

For $K n>10^{-3}$ and the temperature behind the shock of the order of $10,000 \mathrm{~K}$, the relaxation zone for the vibrational mode is of the order or larger than the flow characteristic size. An impact of the vibrational model is the most pronounced in this case. Unfortunately, by now there are very scarce experimental data available on the flows under such conditions. This is probably connected with the complexity of the simulation of high- 
enthalpy rarefied flows in ground facilities. The real gas effects were mainly examined for high-density flows $\left(K n \sim 10^{-4}-10^{-5}\right)$. The validation of results of computations is therefore rather problernatic.

Since the flow is symmetric, axisymmetric versions of SMILE and MONACO were employed to reduce computational costs. For $K n=0.01$, the number of simulated molecules was about 300,000 , and the number of collision cells was about 100,000 in SMILE and $15 ; 000$ in MONACO. For $K \boldsymbol{n}=0.001$, the number of molecules was about 800,000 in SMILE and $3,000,000$ in MONACO. The number of cells was 250,000 and 15,000 , respectively. Note, subcells were used in MONACO to increase the spatial resolution. The VHS model was used for intermolecular collisions, with parameters taken from [13]. For modeling rotation-translation energy exchange, the discrete Larsen-Borgnakke model [14] was used with variable rotational collision number (energydependent in MONACO and temperature-dependent in SMILE). In order to isolate the impact of molecular vibrations, chemical reactions were disabled in these computations. Diffuse reflection with complete energy and momentum accommodation was assumed for the gassurface interaction.

The calculation of the distribution functions was conducted in specified points along the stagnation line, in the side flow, and in the wake flow. The main goal was to examine the effect of vibrational model in the flow inside the shock layers where a strong nonequilibrium between translational/rotational and vibrational modes is observed, in the boundary layer, and in the expansion flow behind a sphere. The results for three points are presented in this paper, namely, $\mathrm{X}=-0.0075, \mathrm{Y}=0$ (Point 1, stagnation line), $\mathrm{X}=0.1, \mathrm{Y}=0.102$ (Point 2, side flow), and $X=0.2, Y=0.1$ (Point 3, wake flow). The sphere center is located at $\mathrm{X}=0.1, \mathrm{Y}=0$.

\section{$5 \quad$ Results and discussion}

\subsection{Probabilities of VT transitions}

To better understand the influence of the VT energy transfer model, let us consider first the comparison of probabilities of different VT level-to-level transitions for Models 2, 3, and 4. The probabilities of the transition from the first vibrational level to the ground state as function of translational temperature are presented in Fig. 1. There is a very good agreement observed between the probabilities for Models 2 and 3 for the wide range of temperatures from 2,500 to $25,000 \mathrm{~K}$. The reasons for the agreement is that the free parameters of the two models were defined in such a way that the $1 \rightarrow 0$ transition probabilities match to experimental Millikan and White dependence with the corresponding high-temperature corrections. Model 4 does not contain any arbitrary adjustable parameters. In this model, the average vibrational energy transferced in a collision agrees with that for the SSH model [15], but the higher levels and multi-level transitions give the main contribution to the energy transfer. The probabilities of the $1 \rightarrow 0$ transition for Model 4 are significantly smaller than for Models 2 and 3.

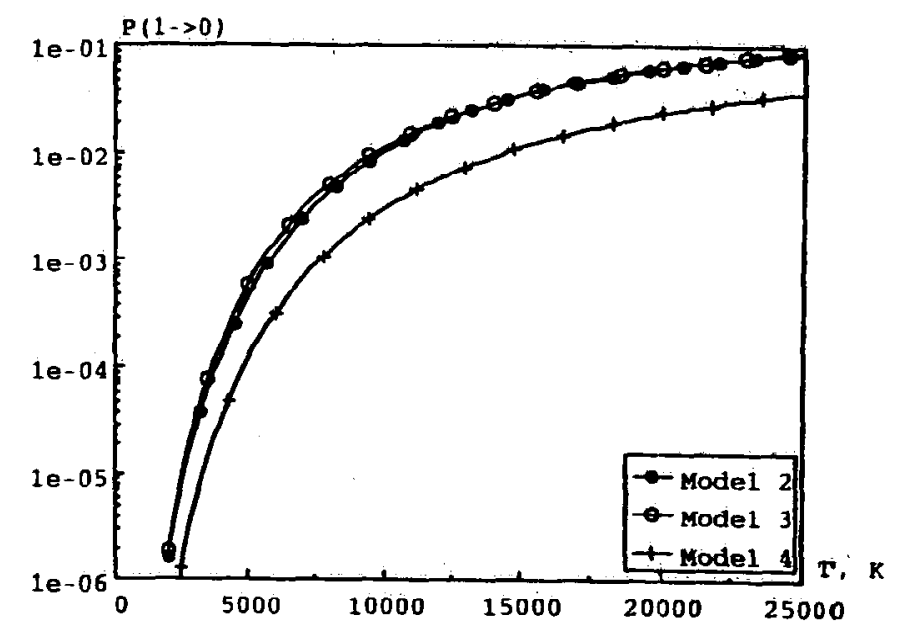

Figure 1: Probabilities of $1 \rightarrow 0$ transition vs. temperature for different models.

The situation changes when considering the probabilities of one-quantum transitions for higher levels. The values for Model 4 approach those for Model 2, while the probabilities for Model 3 are higher than for the other models. This behavior is illustrated in Fig. 2 where $3 \rightarrow 2$ transition probabilities are shown. The difference between Models 2 and 4 slightly decreases for higher temperatures. Generally, the difference between the models is several times larger for relatively low temperatures. However, results of simulations will hardly be affected as the values of probabilities are also small for for these conditions.

Multi-level transitions play an important role at high temperatures since their probabilities increase very rapidly with temperature. A typical example of multilevel transition probabilities as function of temperature is given in Fig. 3, where $3 \rightarrow 0$ probabilities are plotted. In this case, the values for Model 4 overestimate those for Model 3 by a factor of ten at $5,000 \mathrm{~K}$, and the difference even increases when the temperature goes up. Model 2 gives the largest probabilities which are considerably higher than those for the other two models.

As a conclusion of this examination of probabilities, we can say that under the conditions of significant vibration-translation nonequilibrium observed in the bow shock, Model 2 is expected to give faster vibrational relaxation due to higher probabilities of multi- 


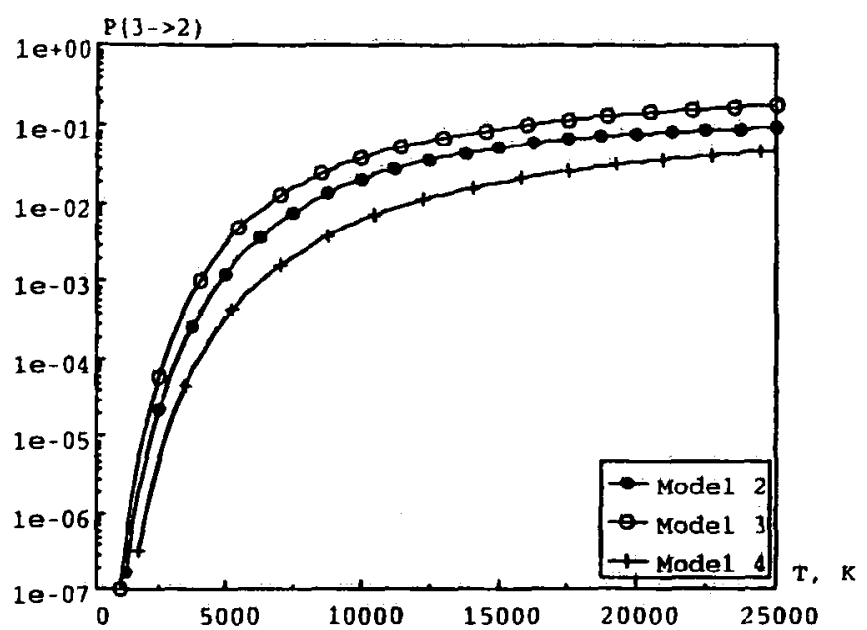

Figure 2: Probabilities of $3 \rightarrow 2$ transition vs. temperature for different models.

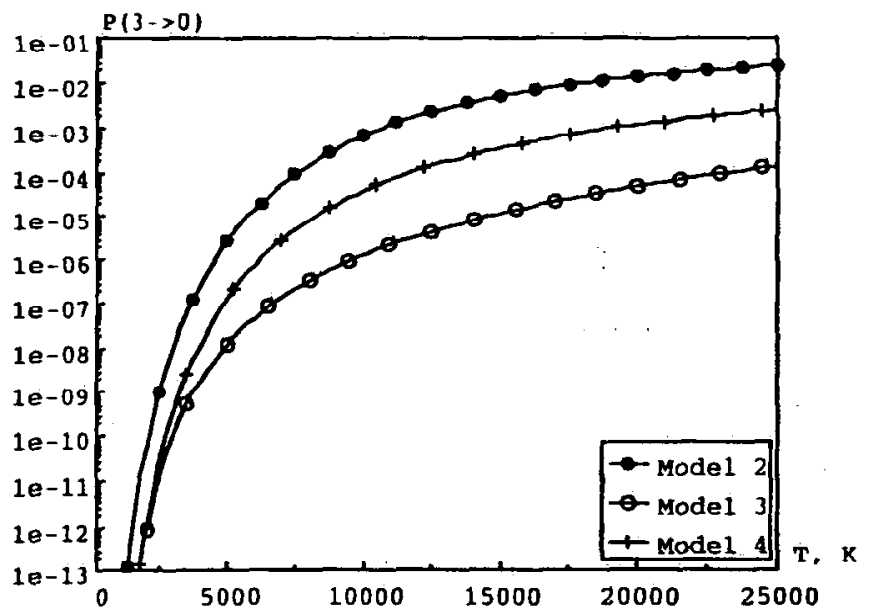

Figure 3: Probabilities of $3 \rightarrow 0$ transition vs. temperature for different models.

level transition. The relation between models 3 and 4 is not clear from the comparison of VT transition probabilities, requiring a direct comparison of calculated temperatures, since a slow VT exchange through one-quantum transitions in Model 3 may compete with a slow multilevel processes of Model 3.

\subsection{The influence of VT model on flow- fields}

Consider now the influence of VT energy transfer models on the macroparameters for two different Knudsen numbers. The computations showed that the impact of the model on all parameters is not significant, except the vibrational temperature. A typical example is shown in Fig. 5.2 where the pressure contours are presented for Models 1 and $3(K n=0.01)$. The isoline val- ues are given in $P a$. It is evident from the figure, that the data agree quite well. There are some differences in the flowfield, especially noticeable near the stagnation line, attributed mainly to a small difference in translational temperatures due to the vibration-translation relaxation.

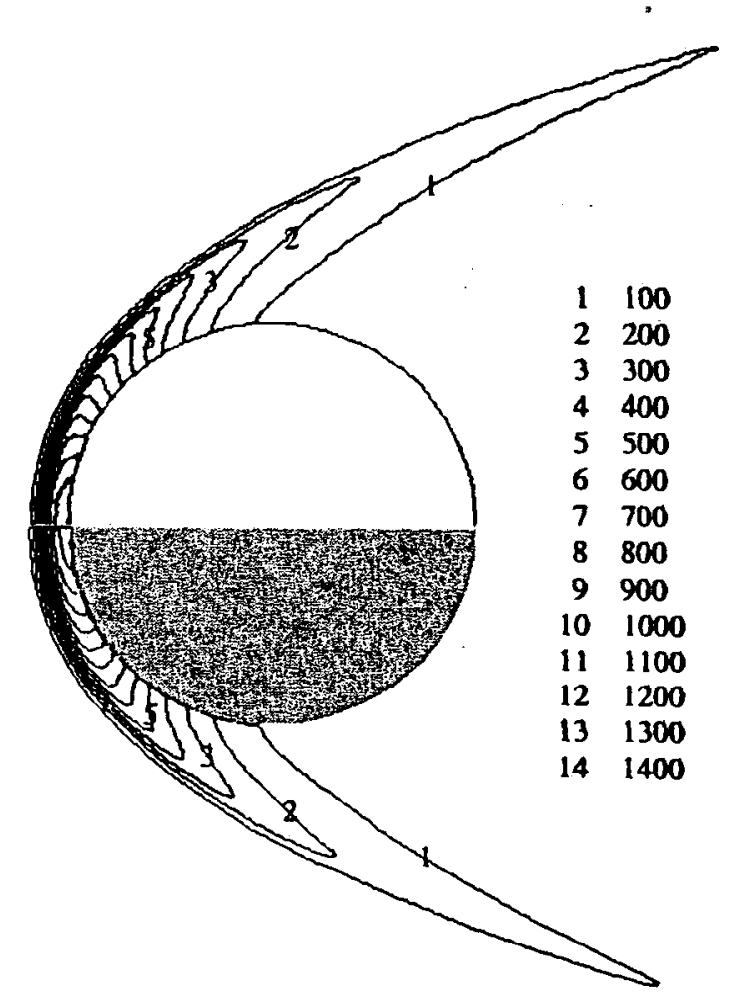

Figure 4: Pressure contours for Model 1 (upper field) and Model 3 (bottom field). $K n=0.01$.

Comparison of vibrational temperatures for these models is presented in Fig. 5. Here and below, isoline values are given in Kelvin. The shock front is slightly thicker for Model 3, while the maximum values in the shock are considerably higher for Model 1. The vibrational temperature values are larger for Model 1 not only in the shock front, but also in the region of expansion flow behind the sphere.

To give a quantitative comparison of vibrational temperatures, the profiles of this property along the stagnation line are presented in Fig. 6 for all four models of VT energy transfer. The abscissa is the distance along the stagnation line in meters, and the stagnation point corresponds to $X=0$. Surprisingly, the data for Model 1 agree very well with those for Model 2, and the profile for Model 3 is close to that of Model 4. The vibrational relaxation of the phenomenological model 1 agrees therefore with Model 2 even under highly nonequilibrium conditions. The agreement of profiles for Mod- 


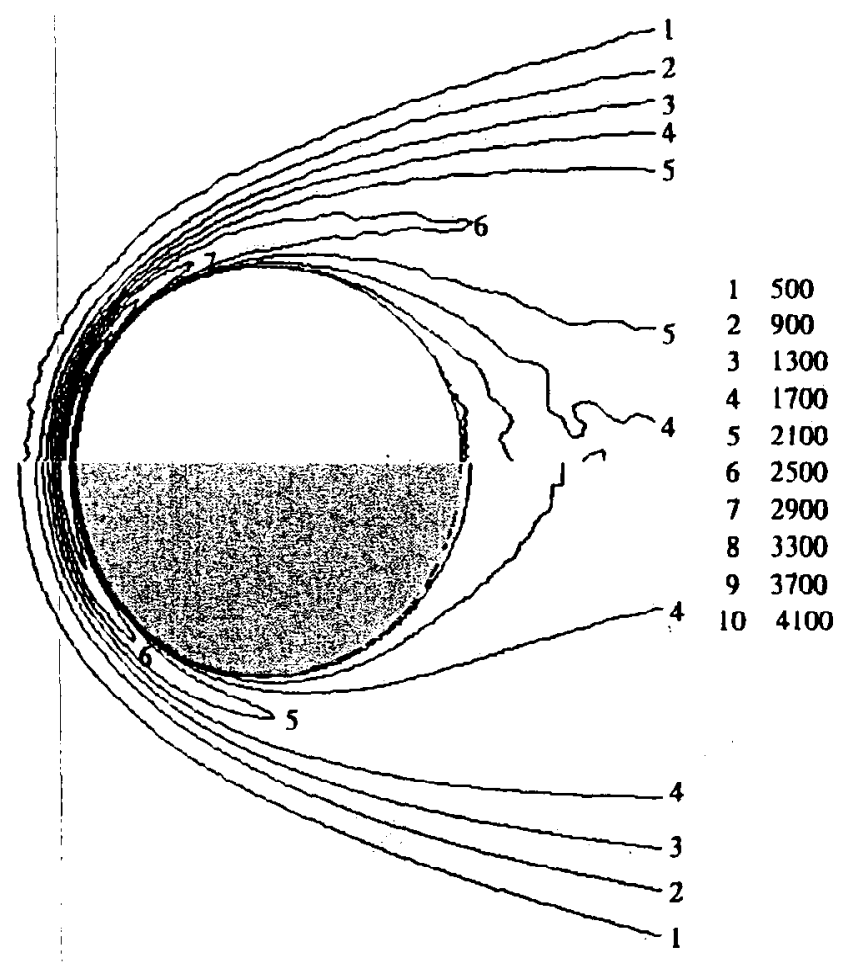

Figure 5: Vibrational temperature contours for Model 1 (upper field) and Model 3 (bottom field). $K n=0.01$.

els 3 and 4 shows that smaller amount of energy transferred from translational to vibrational modes through multi-quantum transitions in model 3 is compensated almost exactly by larger energy transmitted through onequantum transitions. As expected from the comparison of transition probabilities, there is a difference between models 2 and 3,4 . The faster relaxation of the vibrational mode in Model 2 results in higher values of vibrational temperature in the shock front. The maximum is about forty percent larger for this model. It should be noted that the vibrational temperature profile obtained in the relevant continuum CFD calculations [16] of the flow around a sphere is closer to those for Models 1 and 2.

The impact of the VT model on vibrational temperature is less pronounced for the smaller Knudsen number. The temperature contours for $\boldsymbol{K} \boldsymbol{n}=\mathbf{0 . 0 0 1}$ are presented in Fig. 7. The reason for the smaller difference between the models is the much higher collision rate, reducing vibrational-translational nonequilibrium. Again, as for $K n=0.01$, the temperature for Model 1 is higher than that of Model 3 behind the shock as well as in the expansion region.

The profiles of vibrational temperature along the stagnation line for $K n=0.001$ are given in Fig. 8 for all VT models under consideration. The picture resembles the case of $K n=0.01$ (note, a more detailed scale is used

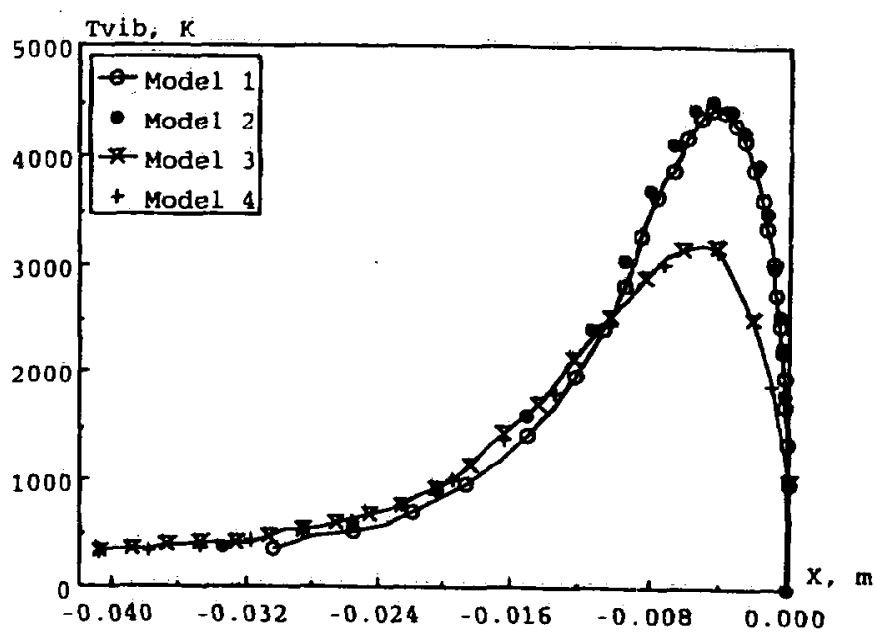

Figure 6: Vibrational temperature along the stagnation line for different models. $K \boldsymbol{n}=0.01$.

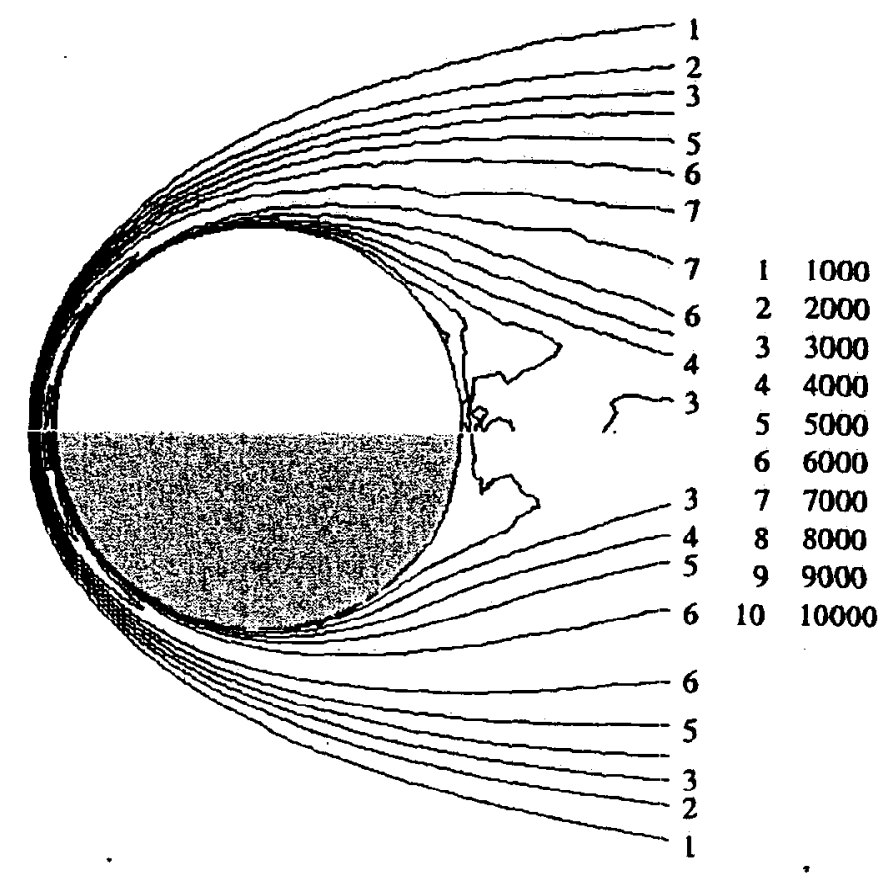

Figure 7: Vibrational temperature contours for Model 1 (upper field) and Model 3 (bottom field). $K n=0.001$.

for $\mathrm{X}$ axis as the shock front is significantly thinner for $K n=0.001$ ). There is a big difference between Models $1 / 2$ and $3 / 4$ in the region near the body. While a higher maximum inside a shock is well explained by the difference in transition probabilities, the reasons for such a difference near the wall are not clear yet, and a more detailed investigation of this problem is needed. 


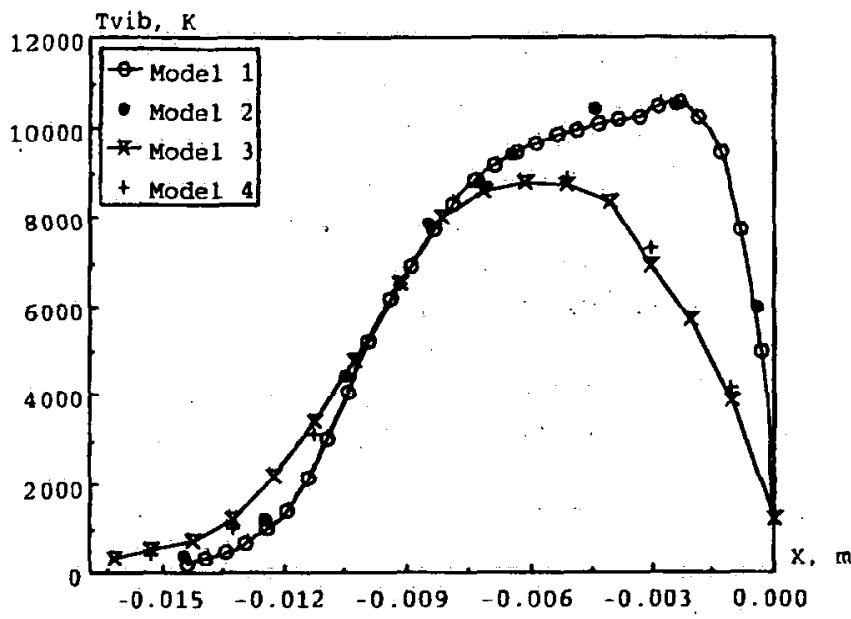

Figure 8: Vibrational temperature along the stagnation line for different models. $K \boldsymbol{n}=\mathbf{0 . 0 0 1}$.

\subsection{VT model effect on vibrational pop- ulations}

In this Section we consider the impact of changing the VT model on vibrational distribution functions in different points of the flow. Note, the computations showed that the translational distribution function for $K n=0.01$ is very close to Maxwellian throughout the flowfield. The rotational distribution is also close to the local equilibrium function, having some minor differences in the shock front. For $K n=0.001$, both translational and rotational distributions essentially coincide with local equilibrium ones.

The comparison of vibrational distributions for $K n=$ 0.01 at a point located inside the shock is presented in Fig. 9 for Models 1 and 2. Since the values of vibrational temperature are very close for these models, only one equilibrium distribution is given here. The equilibrium distribution was calculated using the local vibrational temperature. The major conclusion following from the comparison is that there is a strong nonequilibrium observed for both models. The levels 1,2 , and 3 are underpopulated as compared to the equilibrium function, while the higher levels significantly overpopulated. The shape of the computed function is such that it can be approximated by two lines with different slopes, as though there are two effective vibrational temperatures. The first of them determines the lowest two levels, and the second corresponds to the levels 1 and higher. This behavior is especially evident for Model 1 , with the first effective temperature of about $1,300 \mathrm{~K}$, and the second one, basing on the slope, of approximately $8,000 \mathrm{~K}$. Generally, the definition of vibrational temperature under such nonequilibrium conditions is not clear, making any comparison of vibrational temperatures rather questionable.

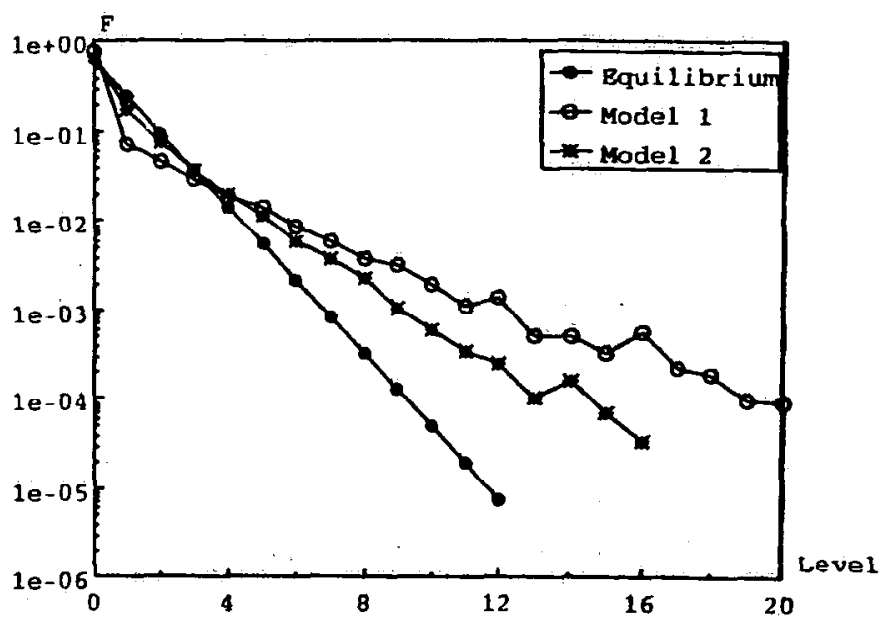

Figure 9: Vibrational distribution function in the shock wave for Models 1 and 2. $K n=0.01$.

The distribution functions for Models 3 and 4 are given in Fig. 10. Again, only one equilibrium function is shown as the vibrational temperatures are close for these models. The shape of the profile for Model 3 can be roughly approximated by two lines. For Model 4, the slope changes gradually from level 0 to level 4 , and the distribution is linear in logarithmic scale only for levels higher than 4. Higher vibrational levels are significantly more densely populated for Model 4.

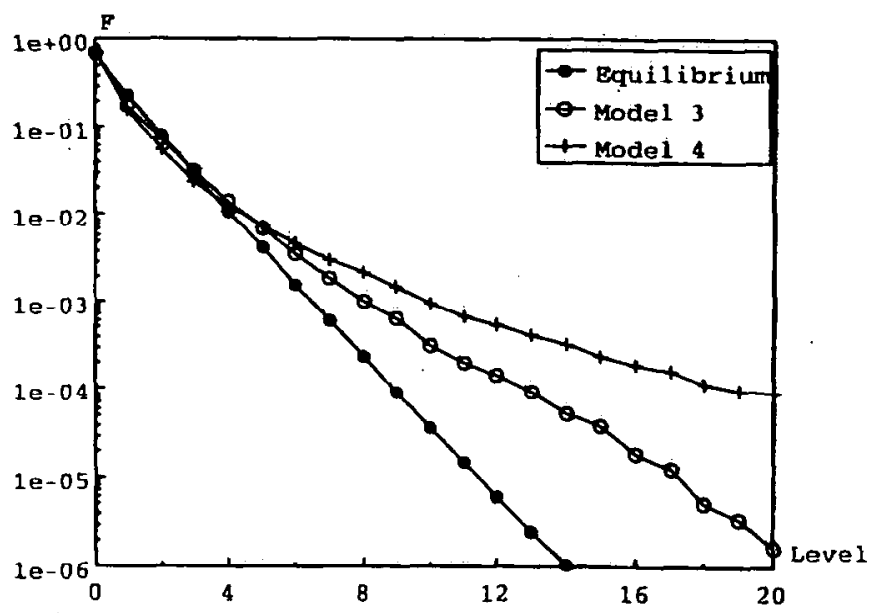

Figure 10: Vibrational distribution function in the shock wave for Models 3 and $4 . K n=0.01$.

Strongly nonequilibrium behavior is therefore observed for all vibrational models inside the shock front, with a relatively large population of high vibrational levels, especially for Models 1 and 4. An oversimplified explanation for that might be the corisideration of two competing molecular fluxes: molecules from the free stream that did not have vibration-translation exchange by the time they reached the cell, and molecules that 
had already have vibrational excitation. The real relaxation process is, of course, more complicated, and the whole system of transition probabilities of the model affects the populations.

Nonequilibrium is also observed in a cell near the wall (Point 2), where the contribution is strong both from vibrationally cold molecules reflected from the body and vibrationally excited molecules from the shock front. The results for this case are given in Figs. 11 and 12. The general behavior of vibrational populations resembles the previous case (a cell inside the shock front), with the difference only in absolute values. The profiles for Models 1 and 2 may be easily approximated by two linear fits, while the slope for the two other models changes more gradually with the increase of vibrational level.

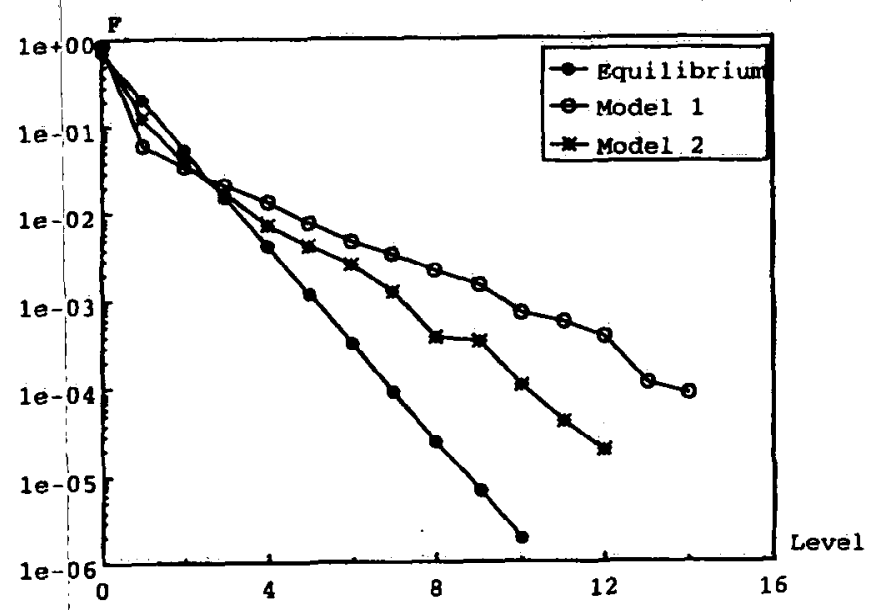

Figure 11: Vibrational distribution function in the boundary layer for Models 1 and 2. $K n=0.01$.

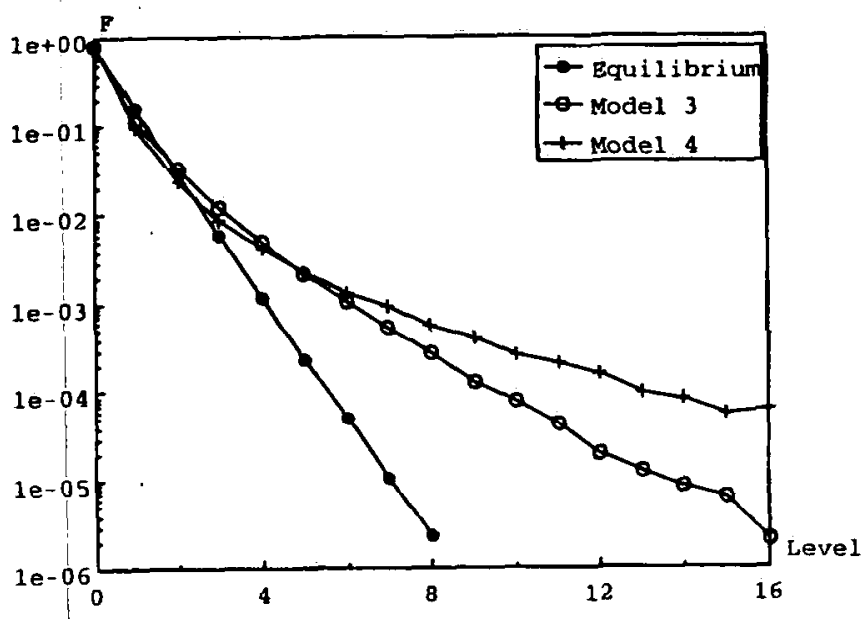

Figure 12: Vibrational distribution function in the boundary layer for Models 3 and $4 . K n=0.01$.

The third point examined was a point in the wake flow. The translational temperature in this region is obviously less then in the shock wave, and the VT relaxation rate is also lower. The population of vibrational levels still has a non-Boltzmann form for all the models, with the underpopulated low levels as compared to the Boltzmann functions, and overpopulated high levels. For the wake flow, there is a smaller difference than was observed between the populations for Models 1 and 2 as well as 3 and 4 , which is attributed to a larger relaxation zone than for the first two regions considered.

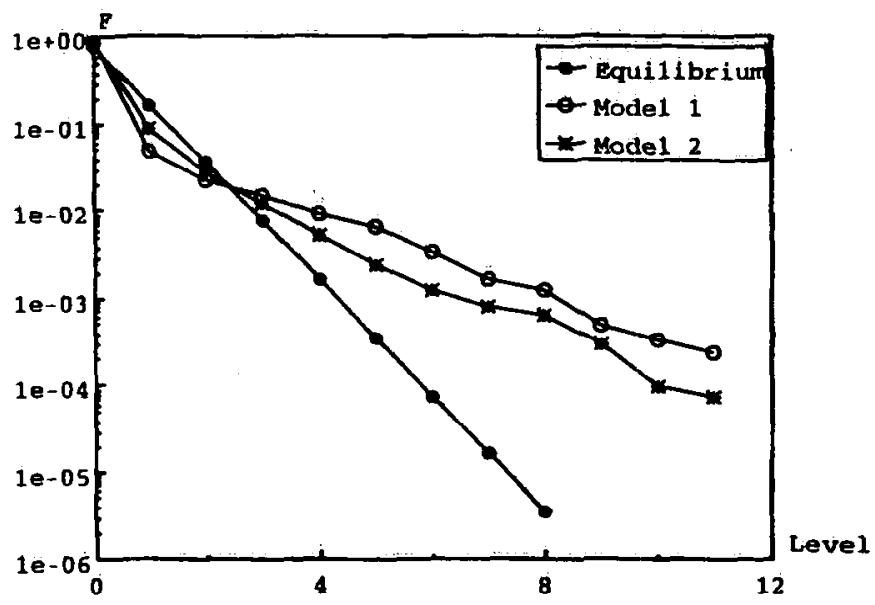

Figure 13: Vibrational distribution function in the expansion flow for Models 1 and 2. $K n=0.01$.

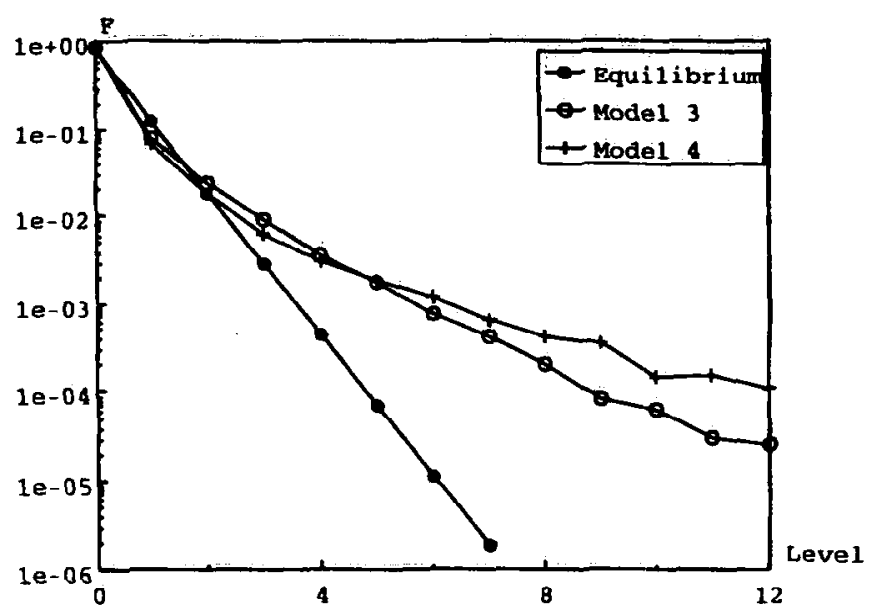

Figure 14: Vibrational distribution function in the expansion flow for Models 3 and 4. $K n=0.01$.

Comparison of vibrational distribution functions for $K n=0.001$ is given in Fig. 15 where the results are presented for Models 1 and 3 and corresponding equilibrium functions in Point 1, i.e. inside the shock front. Even though the degree of nonequilibrium is highest for this region, the high collision frequency results in computed profiles close to the corresponding equilibrium 
data. The difference between computed and equilibrium profiles is larger for Model 3, but it does not exceed a factor of two for high levels. The vibrational populations for Models 2 and 4 are close to those of Models 1 and 3 , and are not shown here. The distribution functions in the regions outside the shock front, such as the boundary layer and expansion flow, agree well with the equilibrium functions.

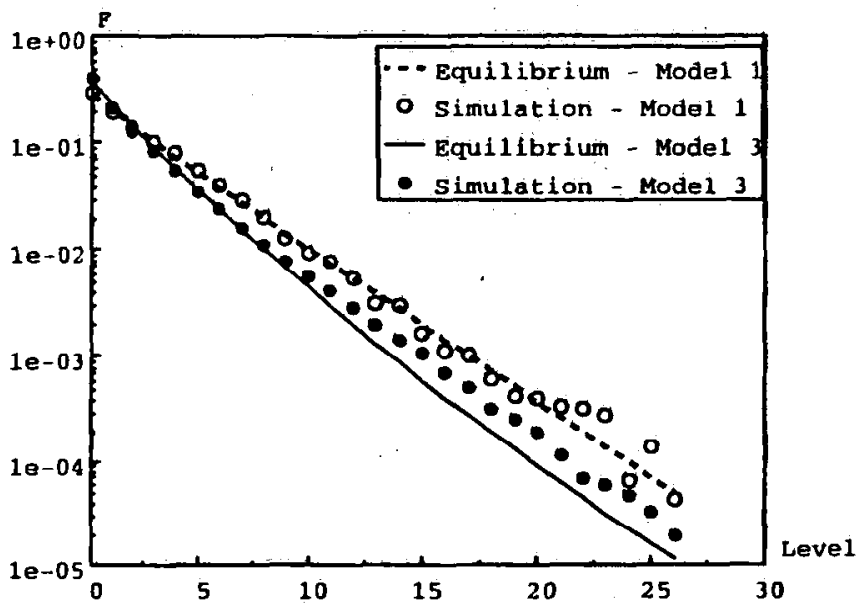

Figure 15: Vibrational distribution function in the shock wave for Models 1 and 3. $K n=0.001$.

\subsection{Effect of VV energy transfer}

The results of the previous section show that there is a strong nonequilibrium in vibrational populations for $K n=0.01$, observed not only in the shock front, but also in the boundary layer and expansion flow region. Four different models were used that accounted for the energy exchange between translational and vibrational modes. Vibration-vibration (VV) transfer in molecular collisions was not included. Since it also may affect vibrational relaxation, the computations were performed where VV transfer was simulated. Model 3 was chosen for calculating VT energy transfer in these computations.

The results for the two cases, with and without VV transfer, are given in Fig. 16 where the vibrational temperature along the stagnation line is shown for $K n=$ 0.01 . It is evident from the figure that there is a very close agreement of the two profiles; meaning the impact of VV energy transfer on vibrational temperature is very small. Obviously, other macroparameters are also not influenced by VV transfer.

While not affecting the macroparameters, the inclusion of VV energy exchange greatly changes the vibrational distribution function, causing their essential equilibration. The comparison of computed vibrational populations with the local equilibrium ones is given in

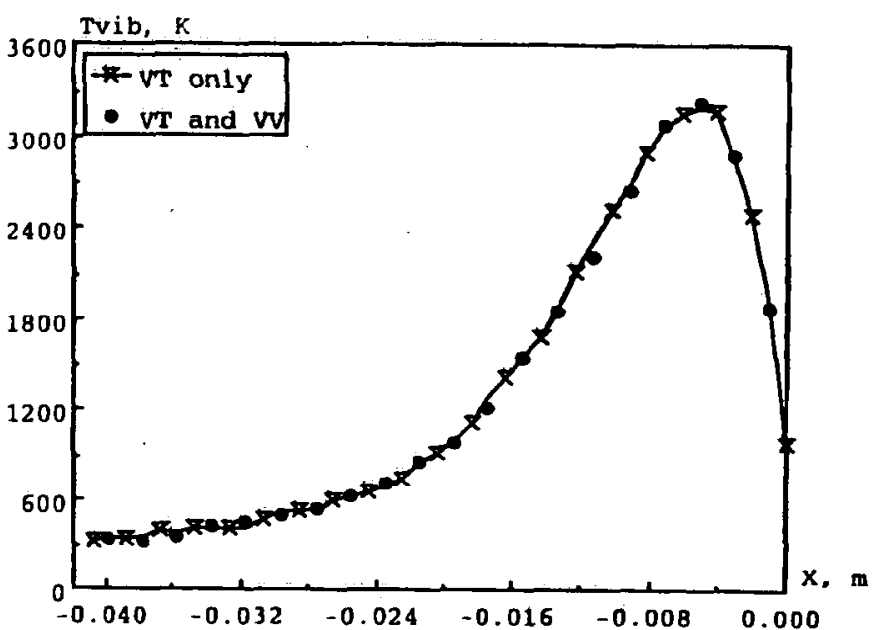

Figure 16: Influence of VV energy transfer on vibrational temperature along the stagnation line.

Figs. 17, 18, and 19, for three different cells. The figures show that taking into account VV energy transfer results in vibrational populations close to equilibrium inside the bow shock (there is a small difference for high levels), and essentially coincident with those in the region of wake flow and in the boundary layer.

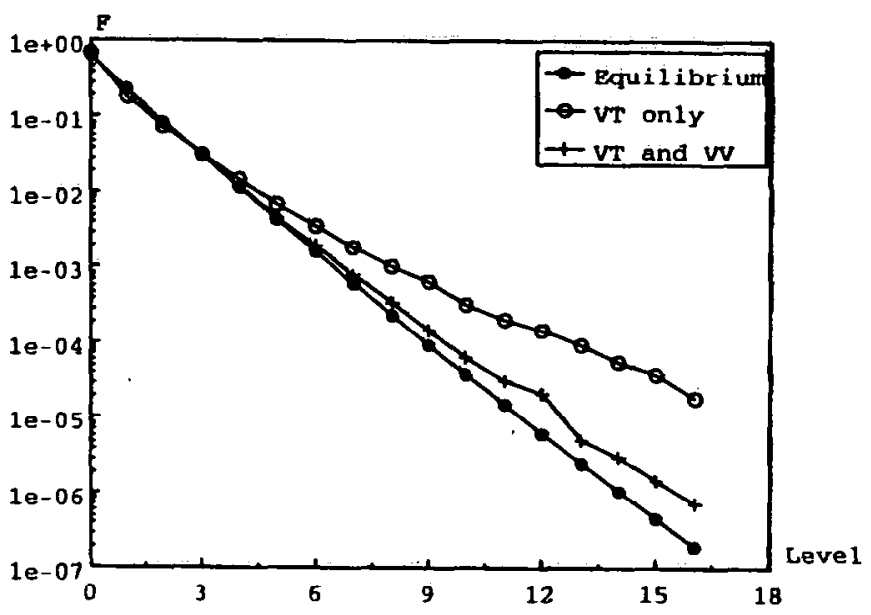

Figure 17: Vibrational distribution function in the shock wave with and without VV transfer for $K n=0.01$.

\section{Conclusions}

Numerical study has been performed to clarify the influence of the model of vibration-translation energy exchange in hypersonic rarefied and near continuum flows. The direct simulations Monte Carlo method was used to calculate a How about a sphere with four different models of vibrational excitation. The computations showed that the change in model of VT energy transfer slightly 


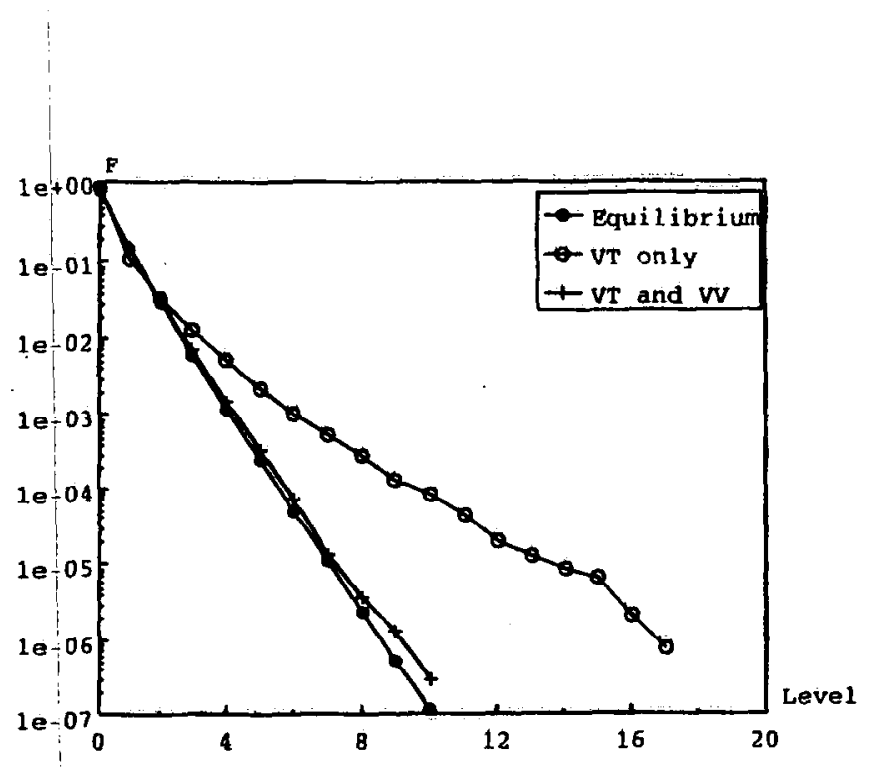

Figure 18: Vibrational distribution function in the boundary layer with and without VV transfer for $K n=$ 0.01 .

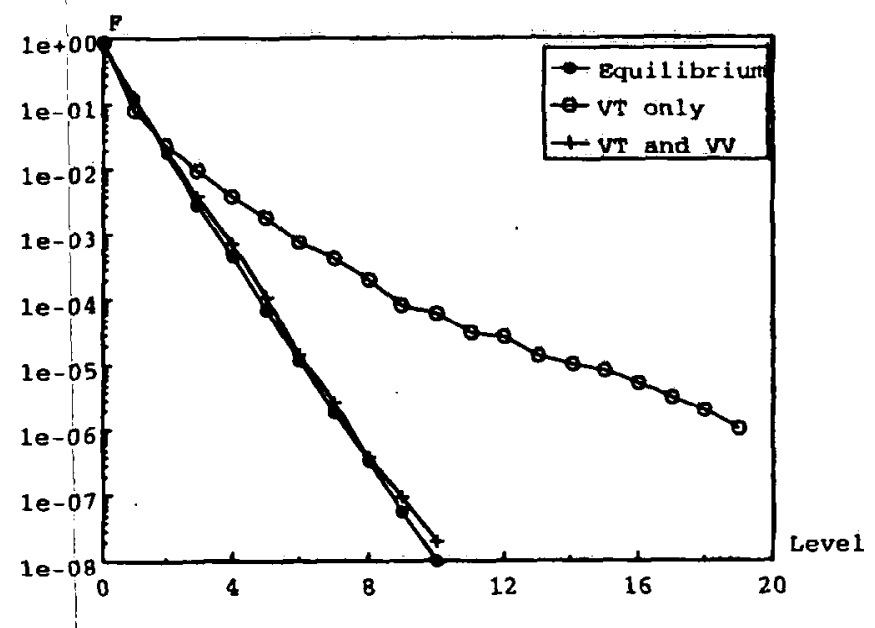

Figure 19: Vibrational distribution function in the expansion flow with and without VV transfer for $K n=$ 0.01 .

changes macroparameters, and has a considerable effect mainly on vibrational temperature. The difference in vibrational temperature in the bow shock amounts to about 40 percent for different models.

For $K n=0.01$ the vibrational distribution function is strongly nonequilibrium not only in the shock front, but also in the boundary layer and expansion flow. Such behavior was observed for all VT models under consideration. For $K n=0.001$, the vibrational populations are close to equilibrium ones even inside the shock front.

Vibration-vibration energy transfer does not affect the macroparameters, but changes significantly the vibrational populations throughout the flowfield. The principal tendency in this case is a fast relaxation of the populations to equilibrium. When accounting for VV transfer, the vibrational distribution function has a
Boltzmann-like shape even for $K n=0.01$.

\section{Acknowledgments}

The authors thank Igor Adamovich for valuable discussions. He also provided a code for calculation of transition probabilities for the nonperturbative semiclassical model. The work at Michigan was funded by the Army Research Office under Grant DAAG55-98-1-0500. The work at Geotge Washington University was supported by the Army Research Office Grant DAAG55-98-1-009 and AFOSR Grant F49620-99-1-0143 and the Ballistic Missile Defense Organization.

\section{References}

[1] Dietrich, S., and Boyd, I.D., "Scalar and parallel optimized implementation of the direct simulation Monte Carlo method," J. Comp. Physics, Vol. 126, No. 2, 1996, pp. 328-342.

[2] Iranov, M.S., Markelov, G.N., and Gimelshein, S.F., "Statistical simulation of reactive rarefied flows: numerical approach and applications," AIAA Pap. No 98-2669, June 1998.

[3] Ivanov, M.S., Markelov, G.N., Gimelshein, S.F., Mishina, L.V., Krylov, A.N., and Grechko, N.V., "High-Altitude Capsule Aerodynamics with Real Gas Effects," Journal of Spacecraft and Rockets, Vol. 35, No. 1, 1998, pp. 16-22.

[4] Vijayakumar, P., Sun, Q., and Boyd, I.D., "Vibrational-Translational Energy Exchange Models For the Direct Simulation Monte Carlo Method," Physics of Fluids, July 1999.

[5] Gimelshein, S.F., Ivanov, M.S., Markelov, G.N., Gorbachev, Yu.E., "Statistical simulation of nonequilibrium rarefied flows with quasiclassical vibrational energy transfer models," Journal of Thermophysics and Heat Transfer, Vol. 12, No. 4, 1998, pp. 2079-2086.

[6] Adamovich, I.V., and Rich, J.W., "ThreeDimensional Nonperturbative Analytic Theory of Vibrational Energy Transfer in Molecule-Molecule Collisions," AIAA Paper 99-3565, June 1999.

[7] Millikan, R.C. and White, R.W, "Systematics of vibrational relaxation," Journal of Chernical Physics, Vol. 39, 1963, p. 3209.

[8] Park, C., "Problems of rate chemistry in the flight regimes of aeroassisted orbital transfer vehicles," 
Progress in Astronautics and Aeronautics: Thermal Design of Aeroassisted orbital transfer vehicles, AIAA, Vol. 96, 1985, p. 511.

[9] Abe, T., Direct simulation Monte Carlo method for internal-translational energy exchange in nonequilibrium flow, Rarefied Gas Dynamics: Theory and Simulations, AIAA, Vol. 159, 1992, p. 103.

[10] Lumpkin, F.E., Haas, B.L. and Boyd, I.D., "Resolution of differences between collision number definitions in particle and continuum simulations," Physics of Fluids A, Vol. 3, 1991, p. 2282.

[11] Kerner, E.H., "Note on the forced and damped oscillator in quantum mechanics," Canadian Journal of Physics, Vol. 36, 1958, p. 371.

[12] Bogdanov, A.V., Dubrovskii, G.V., Gorbachev, Yu.E., and Strelchenya, V.M., "Theory of vibrational and rotational excitation of polyatomic molecules," Physical Reports, Vol. 181, No. 3, pp. 121-206.

[13] Bird, G.A., Molecular Gas Dynamics and the Direct Simulation of Gas Flows, Clarendon Press; Oxford, 1994.

[14] Boyd, I.D., "Relaxation of discrete rotational energy distributions using a Monte Carlo method," Phys. Fluids A, Vol. 5, No. 9, 1993, pp. 2278-2286.

[15] Schwartz, R.N., Slawsky, Z.I., and Herzfeld, K.F., "Calculation of vibrational relaxation times in gases," J. Chem. Physics, Vol. 20, No. 10, 1952, pp. 1591-1599.

[16] Candler, G.V., Olejniczak, J., and Harrold, B., "Detailed simulation of nitrogen dissociation in stagnation regions," Phys. Fluids, Vol. 9, 1997, pp. 21082117. 